\title{
Composite graviton self-interactions in a model of emergent gravity
}

\author{
Christopher D. Carone ${ }^{*}$ and Tangereen V. B. Claringbold ${ }^{\dagger}$ \\ High Energy Theory Group, Department of Physics, College of William and Mary, \\ Williamsburg, Virginia 23187-8795, USA \\ Diana Vaman \\ Department of Physics, University of Virginia, Box 400714, Charlottesville, \\ Virginia 22904, USA
}

(Received 17 November 2017; published 23 July 2018)

\begin{abstract}
We consider a theory of scalars minimally coupled to an auxiliary background metric. The theory is generally covariant and subject to the constraint of vanishing energy-momentum tensor. Eliminating the auxiliary metric leads to a reparametrization invariant, nonpolynomial, metric-independent action for the scalar fields. Working in the limit of a large number of physical scalars, a composite massless spin-2 state, the graviton, was identified in previous work, in a two-into-two scalar scattering process. Here, we further explore the possibility that dynamical emergent gravity is a natural feature of generally covariant quantum field theories, by studying the self-interactions of the emergent composite graviton. We show that the fine-tuning previously imposed to ensure the vanishing of the cosmological constant, as well as the existence of the massless spin-2 state, also assures that the emergent graviton's cubic self-interactions are consistent with those of Einstein's general relativity, up to higher-derivative corrections. We also demonstrate in a theory with more than one type of scalar that the composite graviton coupling is universal.
\end{abstract}

DOI: 10.1103/PhysRevD.98.024041

\section{INTRODUCTION}

In previous work [1], Carone, Erlich, and Vaman presented a theory of $D+N$ scalar fields in which the graviton emerges as a massless composite spin-2 bound state. A number of ingredients were essential as organizing principles in the construction of the model. First, the treelevel action, which included a nonpropagating, auxiliary metric field, was of a form that preserves general covariance. There would be little hope of recovering a massless composite graviton without such a symmetry requirement. For the same reason, the regulator for divergent loop diagrams was required to preserve this symmetry as well. Second, the energy-momentum tensor of the theory was exactly vanishing. Aside from aesthetic simplicity, this condition assures that the emergence of a massless, composite graviton is not in conflict with the Weinberg-

\footnotetext{
*cdcaro@wm.edu

†tvclaringbold@email.wm.edu

\#v3h@virginia.edu
}

Published by the American Physical Society under the terms of the Creative Commons Attribution 4.0 International license. Further distribution of this work must maintain attribution to the author(s) and the published article's title, journal citation, and DOI. Funded by SCOAP ${ }^{3}$.
Witten theorem [2]. It also allows one to solve for the auxiliary metric field and eliminate it from the theory, leading to a nonpolynomial and metric-independent form for the action. When the coordinate invariance of the theory is gauge fixed, $D$ of the scalars, called clock-and-ruler fields in Ref. [1], are gauged away, so that the graviton couples to the nonvanishing energy-momentum tensor of the remaining, physical degrees of freedom (d.o.f.). The main result of Ref. [1] followed from the calculation of a two-into-two scattering amplitude of scalars, to all orders in perturbation theory and at leading order in $1 / N$, where $N \gg 1$ refers to the physical scalar d.o.f. The scattering amplitude was shown to contain a massless pole with an associated Lorentz-index structure consistent with the gauge-invariant part of the graviton propagator. We review the model of Ref. [1] in Sec. II.

The idea that gravity may be the consequence of quantum corrections in a generally covariant nongravitational theory is not new. An early idea due to Sakharov [3] is induced gravity: In this approach, one begins with a quantum field theory (QFT) in a classical background geometry. Upon integrating out the QFT d.o.f., the oneloop partition function will contain the Einstein-Hilbert term for the background metric, a cosmological constant, and higher-order curvature terms. The metric is not quantized, so induced gravity is a semi-classical theory [4]. 
In contrast, the starting point of our emergent gravity scenario is a non-metric QFT. ${ }^{1}$ In Ref. [1], it was shown how the sum over an infinite number of loops in this scalar theory leads to a pole associated with the exchange of a massless spin-2 particle, leading to the identification of the graviton as a composite state. In this paper, we go beyond the linearized order to include the self-coupling of the emergent gravitons. In past models of composite gravitons, going beyond linearized gravity was a notable stumbling block [6,7]. For other related work on gravity as an emergent phenomenon in theories of matter, see Ref. [8].

In the present work, we consider a number of issues that were not resolved in Ref. [1]. The two-into-two scattering calculation of Ref. [1] yielded information on the emergent graviton two-point function, but not on gravitational self interactions. Here we study a scattering amplitude involving six external scalar lines to extract useful information on the emergent three-graviton vertex. We show that the scattering amplitude has the correct form in the limit where it can be compared to the expectations of the weak field expansion of general relativity. As noted in the literature review of Ref. [7], previous composite gravity models have succeeded in achieving a massless spin-2 pole, but have failed to produce the correct graviton self-interactions. Aside from providing a crucial consistency check of Ref. [1], our results represent distinct progress beyond past attempts at constructing consistent models with composite gravitons.

In both the scattering calculations of Ref. [1] and the present work, scalar loops are regulated by dimensional regularization, with a finite regularization parameter $1 / \epsilon$, which we take as a place holder for whatever generally covariant physical regulator may follow from a realistic ultraviolet completion of the theory. As a consequence, the reduced Planck scale $M_{\mathrm{P}}$ was identified in Ref. [1] as a function of $1 / \epsilon$. The same approach is applied in our study of the emergent three-graviton vertex and we show that the identification of $M_{\mathrm{P}}$ in both calculations are in agreement. It is worth stressing that our treatment of the regulator as fixed and determined by a physically measurable quantity is not new, but an approach that is well known in the literature on induced gravity models (see, for example, the review in Ref. [4]). The fact that we employ dimensional regularization rather than, for example, Pauli-Villars fields with a fixed mass scale, is a matter of taste, as any choice that is consistent with general covariance would work equally well. Unlike induced gravity, where the metric is classical, the graviton in our scenario emerges a spin-2 pole in a two-into-two scattering amplitude [1]. An analogous

\footnotetext{
${ }^{1}$ We would like to point out that though we use the term "emergent gravity," this is not the same as the subject of many recent works in which gravity is understood as emerging via entanglement in an underlying microscopic theory (see for example [5]).
}

use of dimensional regularization can also be found in the composite gauge boson models of Ref. [9], where finite $1 / \epsilon$ is related to the value of a measurable coupling. In the present work, we also clarify a technical point that was not explained in Ref. [1]: our calculations follow from a perturbative expansion about a gauge-fixed background configuration for the clock-and-ruler fields. While this implies that our results should maintain a form consistent with the general covariance of the action, it is not clear how exactly this comes about. We show that in our perturbative approach this is due to a cancellation of tree-level and loop effects that is manifest in our study of the three-graviton vertex.

Finally, we touch on two other variations of the original scenario. In the first, we consider a simple extension of the theory in which there are two distinct sets of physical scalars with differing mass, to test the universality of the graviton couplings. We show that in this case that the graviton pole persists, and that the graviton couples to the energy-momentum tensor for each set of scalars with a common Planck mass that depends on all the parameters of the theory. In the second, we show how the effects of background matter can be taken into account by coupling the composite graviton operator to a classical background source, so that the graviton acquires a nonzero vacuum expectation value. Duff [10] showed in the context of general relativity how the presence of the background source modifies the flat-space metric in a way that is consistent with a desired curved background, and demonstrated this by reproducing the mass-expansion of the Schwarzschild metric. We identify the Feynman diagrammatic expansion in the present model that reproduces the expectation value for the graviton that is relevant in this approach.

Our paper is organized as follows. In the next section, we summarize the model of Ref. [1], and correct a number of sign errors that are important in understanding how general covariance is maintained in our perturbative approach. In Sec. III, we isolate the scalar interactions that are relevant for generating the emergent three-graviton coupling via loop effects. In Sec. IV, the loop calculations are performed and the results are compared with the expectations of the weak-field expansion of general relativity. In Sec. V, we consider the extension of the theory to more than one set of distinct scalars and demonstrate the universality of the graviton couplings. In Sec. VI, we discuss one approach to incorporating curved background into the theory. In Sec. VII, we summarize our conclusions.

\section{THE MODEL, WITH SIGNS CORRECTED}

Our starting point is the same as that of Ref. [1]. For the sake of completeness we recall here the main features of the theory we are studying. We are also using this opportunity to correct some sign errors in Ref. [1]. We begin by considering a theory of $N+D$ scalar fields in a curved 
$D$-dimensional spacetime described by a background metric $g_{\mu \nu}$, where $\mu, \nu \in\{0,1, \ldots, D-1\}$ :

$$
\begin{aligned}
S= & \int d^{D} x \sqrt{|g|}\left[\frac { 1 } { 2 } g ^ { \mu \nu } \left(\sum_{a=1}^{N} \partial_{\mu} \phi^{a} \partial_{\nu} \phi^{a}\right.\right. \\
& \left.\left.+\sum_{I, J=0}^{D-1} \partial_{\mu} X^{I} \partial_{\nu} X^{J} \eta_{I J}\right)-V\left(\phi^{a}\right)\right],
\end{aligned}
$$

where $g=\operatorname{det}\left(g_{\mu \nu}\right), g^{\mu \nu}$ is the inverse of the metric $g_{\mu \nu}$, and $\eta_{I J}$ are constants which we take to have the values of the Minkowski metric in $D$ dimensions. (We use the mostlyminus convention for the signature of $\eta_{I J}$ and the metric $g_{\mu \nu}$.) The defining feature of the action in Eq. (2.1) is that it is invariant under general coordinate transformations, with the fields $X^{I}$ and $\phi^{a}$ transforming as scalars and the background field $g_{\mu \nu}$ transforming as a metric tensor. The background metric $g_{\mu \nu}$ has no dynamics, i.e., there is no Einstein-Hilbert term in the action. The theory is defined via functional integration over field configurations subject to the constraint of vanishing energy-momentum tensor; the partition function may be written

$$
Z=\int_{T_{\mu \nu}=0} \mathcal{D} g_{\mu \nu} \mathcal{D} \phi^{a} \mathcal{D} X^{I} e^{i S\left[\phi^{a}, X^{I}, g_{\mu \nu}\right]},
$$

where the energy-momentum tensor is given by

$$
\begin{aligned}
T_{\mu \nu}(x) & =\frac{2}{\sqrt{|g|}} \frac{\delta S}{\delta g^{\mu \nu}(x)} \\
& =\sum_{a=1}^{N} \partial_{\mu} \phi^{a} \partial_{\nu} \phi^{a}+\sum_{I, J=0}^{D-1} \partial_{\mu} X^{I} \partial_{\nu} X^{J} \eta_{I J}-g_{\mu \nu} \mathcal{L},
\end{aligned}
$$

and where the Lagrangian $\mathcal{L}$ is defined by the action in Eq. (2.1), $S \equiv \int d^{D} x \sqrt{|g|} \mathcal{L}$. An explicit solution to $T_{\mu \nu}(x)=0$ for the metric is

$g_{\mu \nu}=\frac{D / 2-1}{V\left(\phi^{a}\right)}\left(\sum_{a=1}^{N} \partial_{\mu} \phi^{a} \partial_{\nu} \phi^{a}+\sum_{I, J=0}^{D-1} \partial_{\mu} X^{I} \partial_{\nu} X^{J} \eta_{I J}\right)$,

which allows the elimination of $g_{\mu \nu}$ from the action upon performing the $\mathcal{D} g_{\mu \nu}$ integration. With the spacetime metric determined by Eq. (2.5), the action for the theory resembles the Dirac-Born-Infeld action with vanishing gauge field, modulated by the scalar-field potential function $V\left(\phi^{a}\right)$ :

$$
\begin{aligned}
S= & \int d^{D} x\left(\frac{\frac{D}{2}-1}{V\left(\phi^{a}\right)}\right)^{\frac{D}{2}-1} \\
& \times \sqrt{\left|\operatorname{det}\left(\sum_{a=1}^{N} \partial_{\mu} \phi^{a} \partial_{\nu} \phi^{a}+\sum_{I, J=0}^{D-1} \partial_{\mu} X^{I} \partial_{\nu} X^{J} \eta_{I J}\right)\right|} .
\end{aligned}
$$

The general coordinate transformation invariance of Eq. (2.1) translated into reparametrization invariance of Eq. (2.6). While Eq. (2.1) is nongeneric (i.e., one could imagine adding other possible terms that are generally covariant), it is the simplest starting point for defining a benchmark model, and hence the most likely to render the extraction of the emergent graviton self-interactions tractable. A result in agreement with Einstein's gravity would support the notion that the same would be obtained in a more complicated theory that is also generally covariant. We proceed next to gauge fix by identifying the clock-andruler fields $X^{I}$ with the corresponding spacetime coordinates, in analogy with the static gauge condition in string theory, up to an overall constant factor,

$$
X^{I}=x^{\mu} \delta_{\mu}^{I} \sqrt{\frac{V_{0}}{\frac{D}{2}-1}-c_{1}}, \quad I=0, \ldots, D-1,
$$

where $c_{1}$ is a counterterm whose role will be revisited below. In order to analyze the theory perturbatively, we write $V(\phi)=V_{0}+\Delta V\left(\phi^{a}\right)$ and expand the action Eq. (2.6) in powers of $1 / V_{0}$. We also assume that $N$, the number of fields $\phi^{a}$ in the theory, is large and keep only leading terms in a $1 / N$ expansion. In the two-into-two scattering calculation of Ref. [1], this made the desired diagrammatic resummation possible.

The gauge-fixed action, expanded to second order in $1 / V_{0}$, reads:

$$
\begin{aligned}
S= & \int d^{D} x\left\{\frac{V_{0}}{D / 2-1}+\frac{1}{2} \sum_{a=1}^{N} \partial_{\mu} \phi^{a} \partial^{\mu} \phi^{a}-\Delta V\left(\phi^{a}\right)\right. \\
& -\frac{\frac{D}{2}-1}{4 V_{0}}\left[\sum_{a=1}^{N} \partial_{\mu} \phi^{a} \partial_{\nu} \phi^{a} \sum_{b=1}^{N} \partial^{\mu} \phi^{b} \partial^{\nu} \phi^{b}\right. \\
& \left.-\frac{1}{2}\left(\sum_{a=1}^{N} \partial_{\mu} \phi^{a} \partial^{\mu} \phi^{a}\right)^{2}\right]-\frac{\frac{D}{2}-1}{2} \frac{\Delta V\left(\phi^{a}\right)}{V_{0}} \sum_{a=1}^{N} \partial_{\mu} \phi^{a} \partial^{\mu} \phi^{a} \\
& \left.+\frac{D}{4} \frac{\left(\Delta V\left(\phi^{a}\right)\right)^{2}}{V_{0}}+\mathcal{O}\left(\frac{1}{V_{0}^{2}}\right)\right\} .
\end{aligned}
$$

We further assume a free theory with $\mathrm{O}(N)$-symmetric potential

$$
\Delta V\left(\phi^{a}\right)=\sum_{a=1}^{N} \frac{m^{2}}{2} \phi^{a} \phi^{a}-c_{2},
$$

The counterterm $c_{1}$ from Eq. (2.7) is chosen is to effectively normal order every occurrence of $\partial_{\mu} \phi^{a} \partial_{\nu} \phi^{a}$ in Eq. (2.8) (i.e., any loop which can be constructed by contracting the two $\phi^{a}$ 's in $\partial_{\mu} \phi^{a} \partial_{\nu} \phi^{a}$ is rendered zero by adding the counterterm $-c_{1} \eta_{\mu \nu}$ ). Also, the role of the counterterm $c_{2}$ from Eq. (2.9) is to normal order every occurrence of $\phi^{a} \phi^{a}$ (i.e., any loop which can be constructed 

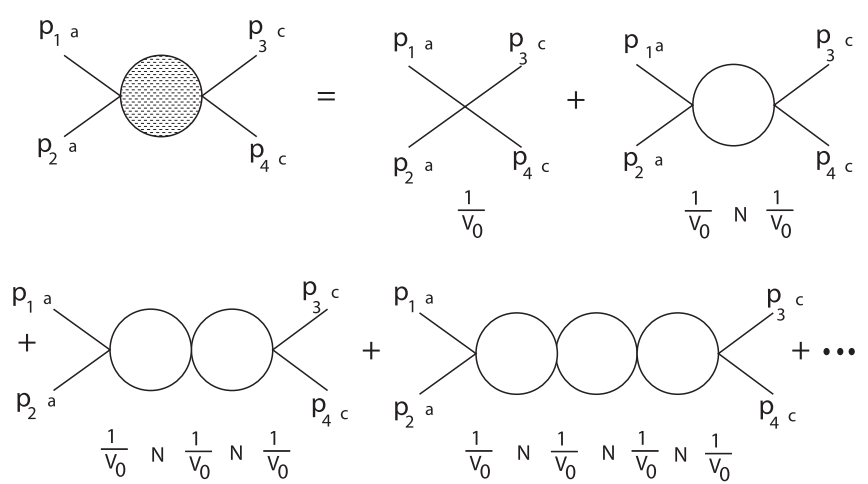

FIG. 1. The scattering amplitude considered in Ref. [1].

by contracting the two $\phi^{a}$ 's in $m^{2} \phi^{a} \phi^{a}$ is canceled by the counterterm $c_{2}$ ).

The graviton pole was identified in Ref. [1] by considering the two-to-two scattering of $\left(\phi^{a} \phi^{a} \rightarrow \phi^{b} \phi^{b}\right)$ scalars in the large- $N$ limit $^{2}$ as shown in Fig. 1. Dimensional regularization was used as a regulator of the loop integrals. The existence of a massless spin-two state (the graviton) being exchanged in this process required the fine-tuned choice ${ }^{3}$

$$
V_{0}=-\frac{N(D / 2-1)}{2} \frac{\Gamma(-D / 2)}{(4 \pi)^{D / 2}}\left(m^{2}\right)^{D / 2},
$$

leading to the following expression for the scattering amplitude:

$$
\begin{aligned}
& A^{\mu \nu \mid \rho \sigma}(q) \\
& =-\frac{3 m^{2}}{D V_{0}}\left[\left(\frac{D}{2}-1\right)\left(\eta^{\nu \rho} \eta^{\mu \sigma}+\eta^{\nu \sigma} \eta^{\mu \rho}\right)-\eta^{\mu \nu} \eta^{\rho \sigma}\right] \frac{1}{q^{2}}+\cdots,
\end{aligned}
$$

where we correct a sign error in Eq. (3.20) of Ref. [1], which propagated into Eq. (3.22) of that paper, and where the ... indicate terms which vanish as $q$ goes on-shell (i.e., $q^{2} \rightarrow 0$ ). The definition of $A^{\mu \nu \mid \rho \sigma}(q)$ is reviewed in the next section. Comparison with the corresponding graviton-mediated scattering amplitude in a free scalar theory ${ }^{4}$

$$
\begin{aligned}
& A^{\mu \nu \mid \rho \sigma}(q) \\
& =-\frac{M_{\mathrm{P}}^{2-D}}{D-2}\left[\left(\frac{D}{2}-1\right)\left(\eta^{\nu \rho} \eta^{\mu \sigma}+\eta^{\nu \sigma} \eta^{\mu \rho}\right)-\eta^{\mu \nu} \eta^{\rho \sigma}\right] \frac{1}{q^{2}},
\end{aligned}
$$

\footnotetext{
${ }^{2}$ This approach is similar to that employed by Suzuki [9] who identified a composite gauge boson in a particular scattering process in a theory of emergent electromagnetism.

${ }^{3}$ Here we correct a sign in the kernel $K^{\lambda \kappa}{ }_{\rho \sigma}$ defined in Eq. (3.16) of Ref. [1]. With $\lambda$ defined in the same way as in Ref. [1], this sign changes the condition for the existence of a pole to $1+\lambda=1+N(D / 2-1) \Gamma(-D / 2)\left(m^{2}\right)^{D / 2} /\left(2 V_{0}(4 \pi)^{D / 2}\right)=0$, yielding Eq. (2.10) above.

${ }^{4}$ Here we correct yet another sign in the scattering amplitude given by Eq. (3.26) of [1].
}

where $M_{\mathrm{P}}$ is the $D$-dimensional Planck mass, leads to

$$
M_{\mathrm{P}}=m\left[\frac{N \Gamma\left(1-\frac{D}{2}\right)}{6(4 \pi)^{D / 2}}\right]^{1 /(D-2)} .
$$

With $D=4-\epsilon$, positivity of the Planck mass requires the regulator $\epsilon$ to be small and negative. The dimensionful constant $V_{0}$ as identified in Eq. (2.10) is however positive. For completeness we revisit the gauge choice Eq. (2.7). Since $c_{1}$ is defined such that $c_{1} \eta_{\mu \nu}$ cancels any loop originating from $\partial_{\mu} \phi^{a} \partial_{\nu} \phi^{a}$, expressing $c_{1}$ in terms of $V_{0}$ leads to

$$
c_{1}=\frac{V_{0}}{\frac{D}{2}-1},
$$

which differs by a sign relative to its expression given in footnote 6 of Ref. [1]. Interestingly, the value of $c_{1}$ that eliminates tadpole diagrams exactly cancels the tree-level gauge choice for the $X^{I}$ in Eq. (2.7).

\section{CONTRIBUTIONS TO THE THREE- GRAVITON COUPLING}

In Ref. [1], the existence of a propagating graviton was demonstrated nonperturbatively, at leading order in a $1 / N$ expansion, by considering the infinite set of diagrams shown in Fig. 1. The scattering amplitude was written

$$
\begin{aligned}
& i \mathcal{M}\left(p_{1}, a ; p_{2}, a \rightarrow p_{3}, c ; p_{4}, c\right) \\
& \quad \equiv E_{\mu \nu}\left(p_{1}, p_{2}\right)\left[i A^{\mu \nu \mid \rho \sigma}(q)\right] E_{\rho \sigma}\left(p_{3}, p_{4}\right),
\end{aligned}
$$

with

$E_{\mu \nu}\left(p_{1}, p_{2}\right) \equiv-\left(p_{1}^{\mu} p_{2}^{\nu}+p_{1}^{\nu} p_{2}^{\mu}\right)+\eta^{\mu \nu}\left(p_{1} \cdot p_{2}+m^{2}\right)$,

and where $q=p_{1}+p_{2}=p_{3}+p_{4}$. The factor $E_{\mu \nu}$ corresponds to the Feynman rule for the flat-space energymomentum tensor for the noninteracting scalar fields,

$\mathcal{T}^{\mu \nu}=\sum_{a=1}^{N}\left[\partial^{\mu} \phi^{a} \partial^{\nu} \phi^{a}-\eta^{\mu \nu}\left(\frac{1}{2} \partial^{\alpha} \phi^{a} \partial_{\alpha} \phi^{a}-\frac{1}{2} m^{2} \phi^{a} \phi^{a}\right)\right]$.

The gauge-invariant part of the graviton propagator was extracted by studying the $1 / q^{2}$ pole in $A^{\mu \nu \mid \rho \sigma}(q)$, and was shown to have the appropriate form. The calculation depended only on the quartic scalar interaction vertex in the theory,

$$
\mathcal{L}_{\text {int }}=-\frac{1}{4 V_{0}} \mathcal{T}_{\mu \nu} \mathcal{T}_{\alpha \beta} P^{\mu \nu \mid \alpha \beta},
$$

where we defined the tensor structure

$P^{\alpha \beta \mid \lambda \kappa} \equiv \frac{1}{2}\left[\left(\frac{D}{2}-1\right)\left(\eta^{\alpha \lambda} \eta^{\beta \kappa}+\eta^{\alpha \kappa} \eta^{\beta \lambda}\right)-\eta^{\lambda \kappa} \eta^{\alpha \beta}\right]$. 


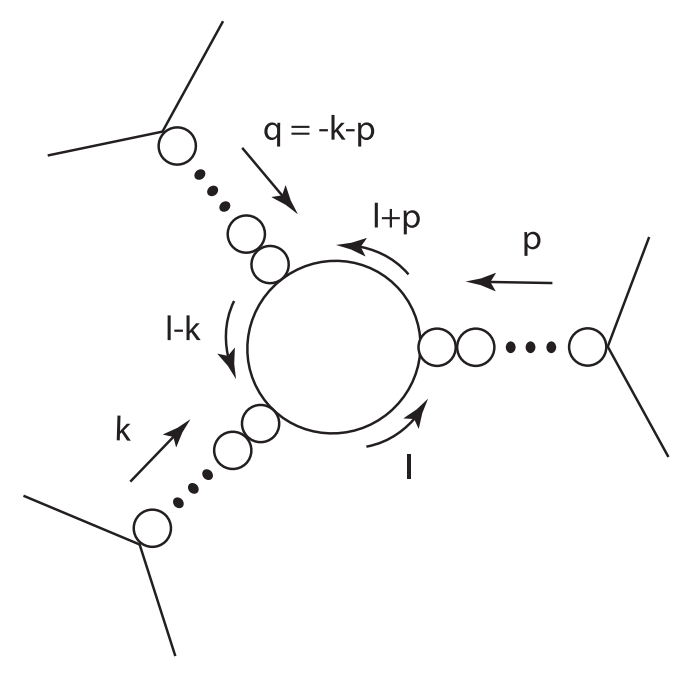

FIG. 2. Contribution to the loop-generated three-graviton coupling involving the quartic scalar interaction vertex exclusively. The chains of small circles corresponds to the sum over loops defined in Fig. 1, which leads to graviton poles. Each pair of external scalars is assumed to be distinct.

At either end of the sum of scattering diagrams in Fig. 1, there are two possible ways of choosing the $\mathcal{T}_{\kappa \lambda}$ factor in Eq. (3.4) that corresponds to the external scalar lines; if we include this combinatoric factor, we may write an effective interaction that represents the coupling of the graviton to two scalars,

$$
\mathcal{L}_{\text {eff }}=-\frac{1}{2} h_{\alpha \beta}^{(1)} \mathcal{T}^{\alpha \beta}
$$

where $h_{\alpha \beta}^{(1)}=\mathcal{T}^{\mu \nu} P_{\mu \nu \mid \alpha \beta} / V_{0}$. (The meaning of the superscript will be explained below.) Equation (3.6) is of the same form as the graviton-scalar-scalar coupling that one would find in a linearized theory with a fundamental graviton field, up to the normalization of the field. However, $-h_{\alpha \beta}^{(1)} / 2$ here represents a composite operator that is quadratic in the scalar fields, with a two-point correlation function given by $i A^{\mu \nu \mid \rho \sigma}(q)$. In Ref. [1], this amplitude was found to contain a pole in $q^{2}$ proportional to $i P_{\alpha \beta \mid \mu \nu} / M_{\mathrm{P}}^{2}$ in $D=4$. Thus, one contribution to the emergent three-graviton coupling arises from a central scalar loop that connects three factors of $T^{\mu \nu}$ associated with the effective interaction in Eq. (3.6). This is illustrated in the six-scalar scattering amplitude shown in Fig. 2. The sum of loops representing each "leg" of this diagram was evaluated in Ref. [1] at leading order in an expansion in $q^{2}$ and $1 / N$. Using this result, we can isolate the leading part of the amplitude in Fig. 2 in a similar expansion. After including the additional contributions described below, the final scattering amplitude can be compared to the same quantity computed in general relativity.

Additional contributions to the emergent three-graviton coupling arise from the presence of six-scalar interactions at the next order in $1 / V_{0}$. Using the solution for $g_{\mu \nu}$ that yields a vanishing total energy-momentum tensor, we may write $g_{\mu \nu}=\eta_{\mu \nu}+h_{\mu \nu}$, where

$$
h_{\mu \nu}=h_{\mu \nu}^{(1)}+h_{\mu \nu}^{(2)}+\cdots,
$$

with

$$
h_{\mu \nu}^{(1)}=-\frac{\Delta V}{V_{0}} \eta_{\mu \nu}+\frac{(D / 2-1)}{V_{0}} \partial_{\mu} \phi \cdot \partial_{\nu} \phi \equiv \frac{1}{V_{0}} P^{\alpha \beta}{ }_{\lambda \kappa} \mathcal{T}_{\alpha \beta},
$$

and

$$
h_{\mu \nu}^{(2)}=-\frac{\Delta V}{V_{0}} h_{\mu \nu}^{(1)} .
$$

The second equality in Eq. (3.8) follows after some simple algebra. The operator $h_{\mu \nu}^{(1)}$ is the same as the one identified in our discussion of the graviton-scalar-scalar coupling. The superscript indicates the order in an expansion in $1 / V_{0}$. Using Eqs. (3.8) and (3.9), we expand the action in our theory, Eq. (2.6), to order $1 / V_{0}^{2}$, while expressing the result entirely in terms of $h_{\mu \nu}^{(1)}$ :

$$
\begin{aligned}
\mathcal{L}_{\text {int }}= & \frac{V_{0}}{(D / 2-1)}\left[\frac{1}{6} h^{(1) \alpha}{ }_{\beta} h^{(1) \beta}{ }_{\gamma} h^{(1) \gamma}{ }_{\alpha}-\frac{1}{8} h^{(1)} h^{(1) \alpha}{ }_{\beta}^{(1) \beta}{ }_{\alpha}\right. \\
& \left.+\frac{1}{48} h^{(1) 3}-\frac{\Delta V}{V_{0}}\left(-\frac{1}{4} h^{(1) \alpha} h_{\alpha}^{(1) \beta}+\frac{1}{8} h^{(1) 2}\right)\right] .
\end{aligned}
$$

Each factor of $-h^{(1)} / 2$ in Eq. (3.10) can connect to the sum over loop diagrams, described earlier, that generate graviton poles; the first three terms in Eq. (3.10) lead to an effective three-graviton contact interaction that is independent of momentum. No such interaction exists in general relativity; we will show later how the effects of these terms are cancelled. A separate contribution to the three-graviton coupling arises in a similar way from the remaining terms in Eq. (3.10), except that the factor of $\Delta V$ must be joined to the quartic scalar vertex in Eq. (3.6) by a loop. The contributions to the six-scalar scattering amplitude discussed earlier that follow from the interactions in Eq. (3.10) are shown in Fig. 3. Note that a contribution to the threegraviton coupling arising from the eight-scalar interactions, at order $1 / V_{0}^{3}$, is absent in this theory. Such a diagram would require closing a single scalar loop that is cancelled by the counterterms, appearing in Eqs. (2.7) and (2.9), that remove all the tadpole diagrams in the theory.

We evaluate the diagrams of Figs. 2 and 3 in the next section. We show that the part of the result that is quadratic in the graviton momenta is consistent with the three-graviton coupling of general relativity, and the part that is independent of momentum is vanishing. We verify that the Planck scale determined from this calculation agrees with the result obtained via the scattering amplitude of Ref. [1]. In both 


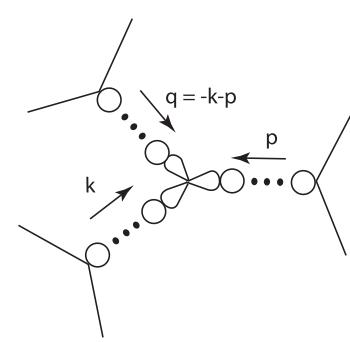

(a)

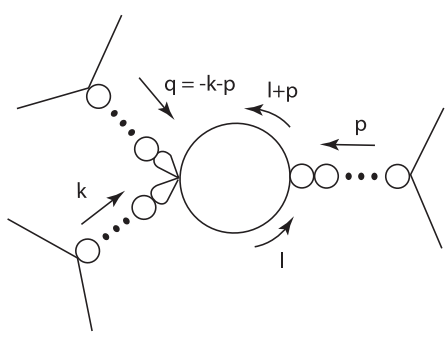

(b)
FIG. 3. Contribution to the loop-generated three-graviton coupling involving six-scalar interaction vertices. The two diagrams shown, labelled (a) and (b), are discussed in the text. The chains of small circles corresponds to the sum over loops defined in Fig. 1, which leads to graviton poles. Each pair of external scalars is assumed to be distinct. Two other diagrams similar to the second one are not shown.

calculations, loops are regulated using dimensional regularization, with $D=4-\epsilon$, and $\epsilon$ fixed at a finite value. This approach is used as a placeholder for whatever generally covariant physical regulator may be provided by a realistic ultraviolet completion of the theory. Proceeding in this way, the Planck scale becomes a function of $1 / \epsilon$ and we will verify that the result obtained in the next section agrees exactly with the one found in Ref. [1].

\section{EMERGENT THREE-GRAVITON VERTEX AND $M_{P}$}

In this section, we study the six-scalar scattering amplitude given by the diagrams shown in Figs. 2 and 3 , and compare to the result expected from general relativity. The chains of circles forming the three legs of the diagrams represent the same sums over loops shown in Fig. 1 that provide the emergent graviton poles. Since these were previously isolated as the leading order terms in an expansion in $q^{2}$, we can reliably compute the leading order piece of the scattering amplitude as the graviton legs are taken nearly on shell. It is convenient to parametrize the result as

$$
i \mathcal{M}=\frac{1}{M_{\mathrm{P}}^{6}} \frac{1}{p^{2}} \frac{1}{q^{2}} \frac{1}{k^{2}} \mathcal{A},
$$

where we define

$$
\mathcal{A}=E_{1}^{\lambda \kappa} E_{2}^{\psi \omega} E_{3}^{\phi \chi} P_{\lambda \kappa \mid \alpha \beta} P_{\psi \omega \mid \gamma \delta} P_{\phi \chi \mid \epsilon \zeta} A^{\alpha \beta \gamma \delta \epsilon \zeta} .
$$

Here, $E_{1}, E_{2}$, and $E_{3}$ refer to the external line factors, defined earlier, that connect to graviton lines carrying momenta $p, k$, and $q$, respectively. Note that we will only need to evaluate the $1 / \epsilon$ dependence of the central loops in the diagrams of Figs. 2 and 3, so that all vertices are evaluated for $D=4$.

The innermost loop in the diagram of Fig. 2 involves three insertions of the energy-momentum tensor $\mathcal{T}^{\mu \nu}$, which has the Feynman rule

$$
i\left[p_{1}^{\mu} p_{2}^{\nu}+p_{1}^{\nu} p_{2}^{\mu}+\eta^{\mu \nu}\left(m^{2}-p_{1} \cdot p_{2}\right)\right]
$$

for momentum $p_{1}$ flowing in and momentum $p_{2}$ flowing out. The amplitude is then given by

$$
\begin{aligned}
& A_{1}^{\alpha \beta \gamma \delta \epsilon \zeta} \\
& \quad=N \int \frac{d^{4} \ell}{(2 \pi)^{4}} \frac{N_{1}^{\alpha \beta \gamma \delta \epsilon \zeta}}{\left[(p+\ell)^{2}-m^{2}\right]\left[\ell^{2}-m^{2}\right]\left[(\ell-k)^{2}-m^{2}\right]},
\end{aligned}
$$

where the numerator factor is

$$
\begin{aligned}
& N_{1}^{\alpha \beta \gamma \delta \epsilon \zeta} \\
& \begin{aligned}
= & {\left[\ell^{\alpha}(\ell+p)^{\beta}+\ell^{\beta}(\ell+p)^{\alpha}+\eta^{\alpha \beta}\left(m^{2}-\ell \cdot(\ell+p)\right)\right] } \\
& \times\left[(\ell+p)^{\epsilon}(\ell-k)^{\zeta}+(\ell+p)^{\zeta}(\ell-k)^{\epsilon}\right. \\
& \left.+\eta^{\epsilon \zeta}\left(m^{2}-(\ell+p) \cdot(\ell-k)\right)\right]\left[(\ell-k)^{\gamma} \ell^{\delta}+(\ell-k)^{\delta} \ell^{\gamma}\right. \\
& \left.+\eta^{\gamma \delta}\left(m^{2}-(\ell-k) \cdot \ell\right)\right],
\end{aligned}
\end{aligned}
$$

and momenta are defined as in Fig. 2. Similarly, diagram of Fig. 3(b) leads to the amplitude

$A_{2}^{\alpha \beta \gamma \delta \epsilon \zeta}=m^{2} N \int \frac{d^{4} \ell}{(2 \pi)^{4}} \frac{N_{2}^{\alpha \beta \gamma \delta \epsilon \zeta}}{\left[(p+\ell)^{2}-m^{2}\right]\left[\ell^{2}-m^{2}\right]}$,

where the numerator factor is

$$
\begin{aligned}
N_{2}^{\alpha \beta \gamma \delta \epsilon \zeta}= & -\left[\ell^{\alpha}(\ell+p)^{\beta}+\ell^{\beta}(\ell+p)^{\alpha}\right. \\
& \left.+\eta^{\alpha \beta}\left(m^{2}-\ell \cdot(\ell+p)\right)\right] P^{\gamma \delta \mid \epsilon \zeta} .
\end{aligned}
$$

Let us first focus on the part of the amplitude that is quadratic in the momenta $p, k$, and $q$, which excludes any contribution from the diagram of Fig. 3(a). We note by inspection of Eq. (4.6) that $A_{2}^{\alpha \beta \gamma \delta \epsilon \zeta}$ will involve terms proportional to either $p^{\alpha} p^{\beta}$ or $p^{2} \eta^{\alpha \beta}$. Terms of the first type vanish since they are contracted with the external line factor $E_{1}$, while terms of the second type are higher-order in our expansion about the limit $p^{2}=k^{2}=q^{2}=0$. Hence, the diagram of Fig. 3(b) does not contribute at leading order to the part of the amplitude $\mathcal{A}$ that is quadratic in the graviton momenta.

The diagram of Fig. 2, however, does contribute. Using Eq. (4.2) and the notation $E_{i}{ }^{\mu}{ }_{\mu} \equiv E_{i}$ and $E_{i \mu \nu} E_{j}^{\mu \nu} \equiv E_{i} \cdot E_{j}$, we find

$$
\begin{aligned}
\mathcal{A}= & \frac{i N}{8 \pi^{2}} \frac{m^{2}}{\epsilon}\left[-\frac{1}{6} E_{1} E_{2} E_{3}^{\mu \nu} k_{\mu} k_{\nu}+\frac{1}{3} E_{1} \cdot E_{2} E_{3}^{\mu \nu} k_{\mu} k_{\nu}\right. \\
& \left.-\frac{2}{3} E_{1}^{\mu \nu} k_{\nu} E_{2}^{\alpha \beta} p_{\beta} E_{3 \mu \alpha}+\text { perms }\right]+ \text { higher order },
\end{aligned}
$$

where "perms" refers to two additional cyclic permutations in which 


$$
\left(E_{1}, p\right) \rightarrow\left(E_{2}, k\right) \rightarrow\left(E_{3}, q\right) \rightarrow\left(E_{1}, p\right)
$$

and "higher order" represents terms that vanish when the gravitons are exactly on shell.

We can compute the analogous result in the weak field expansion of general relativity. We work with a rescaling of the graviton field so that the propagator in de Donder gauge is $i P_{\alpha \beta \mid \mu \nu} / q^{2}$, and the coupling of the graviton to the energy-momentum tensor is $h_{\mu \nu} \mathcal{T}^{\mu \nu} / M_{\mathrm{P}}$. Defining the following quantities,

$$
\begin{gathered}
f_{1}=-i p^{2} \eta_{\alpha \beta} \eta_{\gamma \delta} \eta_{\epsilon \zeta}, \\
f_{2}=-\frac{i}{2} q^{2} \eta_{\alpha \beta}\left(\eta_{\gamma \zeta} \eta_{\epsilon \delta}+\eta_{\delta \zeta} \eta_{\epsilon \gamma}\right), \\
f_{3}=-\frac{i}{2} q \cdot k \eta_{\alpha \beta}\left(\eta_{\gamma \epsilon} \eta_{\delta \zeta}+\eta_{\epsilon \delta} \eta_{\zeta \gamma}\right), \\
f_{4}=-\frac{i}{8} q^{2}\left(\eta_{\beta \epsilon} \eta_{\gamma \zeta} \eta_{\alpha \delta}+\eta_{\beta \epsilon} \eta_{\delta \zeta} \eta_{\alpha \gamma}+\eta_{\beta \zeta} \eta_{\gamma \epsilon} \eta_{\alpha \delta}+\eta_{\beta \zeta} \eta_{\delta \epsilon} \eta_{\alpha \gamma}\right. \\
\left.+\eta_{\alpha \epsilon} \eta_{\gamma \zeta} \eta_{\beta \delta}+\eta_{\alpha \epsilon} \eta_{\delta \zeta} \eta_{\beta \gamma}+\eta_{\alpha \zeta} \eta_{\gamma \epsilon} \eta_{\beta \delta}+\eta_{\alpha \zeta} \eta_{\delta \epsilon} \eta_{\beta \gamma}\right), \\
f_{6}=-i k_{\alpha} k_{\beta} \eta_{\epsilon \zeta} \eta_{\gamma \delta}, \\
f_{7}=-\frac{i}{4}\left(q_{\alpha} k_{\beta}+q_{\beta} k_{\alpha}\right)\left(\eta_{\epsilon \delta} \eta_{\zeta \gamma}+\eta_{\epsilon \gamma} \epsilon_{\zeta \delta}\right) \\
f_{8}=-\frac{i}{2} k_{\alpha} k_{\beta}\left(\eta_{\epsilon \gamma} \eta_{\zeta \delta}+\eta_{\epsilon \delta} \eta_{\zeta \gamma}\right), \\
f_{9}=-\frac{i}{4} \eta_{\alpha \beta}\left(q_{\epsilon} k_{\gamma} \eta_{\delta \zeta}+q_{\epsilon} k_{\delta} \eta_{\gamma \zeta}+q_{\zeta} k_{\gamma} \eta_{\delta \epsilon}+q_{\zeta} k_{\delta} \eta_{\gamma \epsilon}\right),
\end{gathered}
$$

$$
f_{10}=-\frac{i}{4} \eta_{\alpha \beta}\left(q_{\epsilon} q_{\gamma} \eta_{\zeta \delta}+q_{\epsilon} q_{\delta} \eta_{\zeta \gamma}+q_{\zeta} q_{\gamma} \eta_{\epsilon \delta}+q_{\zeta} q_{\delta} \eta_{\epsilon \gamma}\right),
$$

$$
f_{11}=-\frac{i}{4} \eta_{\alpha \beta}\left(k_{\epsilon} q_{\gamma} \eta_{\zeta \delta}+k_{\epsilon} q_{\delta} \eta_{\zeta \gamma}+k_{\zeta} q_{\gamma} \eta_{\epsilon \delta}+k_{\zeta} q_{\delta} \eta_{\epsilon \gamma}\right),
$$

$$
\begin{aligned}
f_{13}= & -\frac{i}{8}\left(q_{\epsilon} q_{\gamma} \eta_{\alpha \zeta} \eta_{\beta \delta}+q_{\epsilon} q_{\delta} \eta_{\alpha \zeta} \eta_{\beta \gamma}+q_{\zeta} q_{\gamma} \eta_{\alpha \epsilon} \eta_{\beta \delta}\right. \\
& +q_{\zeta} q_{\delta} \eta_{\alpha \epsilon} \eta_{\beta \gamma}+q_{\epsilon} q_{\gamma} \eta_{\beta \zeta} \eta_{\alpha \delta}+q_{\epsilon} q_{\delta} \eta_{\beta \zeta} \eta_{\alpha \gamma} \\
+ & \left.q_{\zeta} q_{\gamma} \eta_{\beta \epsilon} \eta_{\alpha \delta}+q_{\zeta} q_{\delta} \eta_{\beta \epsilon} \eta_{\alpha \gamma}\right), \\
f_{14}= & -\frac{i}{8}\left[\left(k_{\epsilon} \eta_{\alpha \zeta}+k_{\zeta} \eta_{\alpha \epsilon}\right)\left(q_{\gamma} \eta_{\beta \delta}+q_{\delta} \eta_{\beta \gamma}\right)\right. \\
& \left.+\left(k_{\epsilon} \eta_{\beta \zeta}+k_{\zeta} \eta_{\beta \epsilon}\right)\left(q_{\gamma} \eta_{\alpha \delta}+q_{\delta} \eta_{\alpha \gamma}\right)\right],
\end{aligned}
$$

we find that the Feynman rule for the three-graviton vertex shown in Fig. 4 can be written

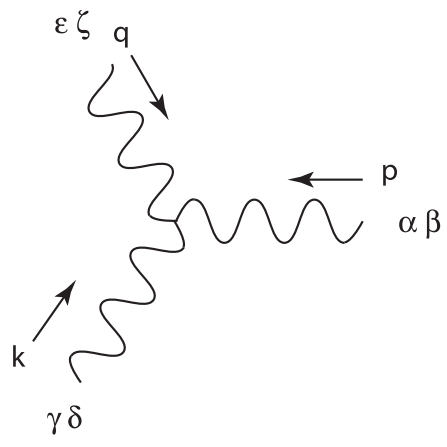

FIG. 4. Conventions for the Feynman rule for the three-graviton vertex.

$$
i V_{3 h}^{\alpha \beta \gamma \delta \zeta}=-\frac{4}{M_{\mathrm{P}}}\left(\sum_{i} c_{i} f_{i}+\text { perms }\right),
$$

where "perms" refers to the five remaining permutations of the labels of the external lines $(\alpha, \beta, p),(\gamma, \delta, k)$, and $(\epsilon, \zeta, q)$. The coefficients $c_{i}$ are given by

$$
\begin{aligned}
& c_{1}=\frac{1}{16}, \quad c_{2}=-\frac{1}{2}, \quad c_{3}=-\frac{3}{8}, \quad c_{4}=\frac{1}{4}, \\
& c_{5}=0, \quad c_{6}=-\frac{1}{4}, \quad c_{7}=\frac{1}{4}, \quad c_{8}=\frac{1}{2}, \\
& c_{9}=\frac{1}{2}, \quad c_{10}=1, \quad c_{11}=\frac{1}{4}, \\
& c_{12}=0, \quad c_{13}=-1, \quad c_{14}=-\frac{1}{2} \text {. }
\end{aligned}
$$

The origin of the $f_{i}$ and the normalization of Eq. (4.22) are described briefly in the Appendix ${ }^{5}$ Computing the same six-scalar amplitude that we considered in our purely scalar theory, we find

$$
\begin{aligned}
\mathcal{A}_{G R}= & -6 i M_{\mathrm{P}}^{2}\left[( k ^ { 2 } + p ^ { 2 } + q ^ { 2 } ) \left(\frac{1}{3} E_{1}^{\mu \nu} E_{2 \nu}{ }^{\alpha} E_{3 \alpha \mu}\right.\right. \\
& \left.+\frac{1}{12} E_{1} E_{2} E_{3}\right)+\left\{-\frac{1}{6} E_{1} E_{2} E_{3}^{\mu \nu} k_{\mu} k_{\nu}+\frac{1}{3} E_{1}\right. \\
& \cdot E_{2} E_{3}^{\mu \nu} k_{\mu} k_{\nu}-\frac{2}{3} E_{1}^{\mu \nu} k_{\nu} E_{2}^{\alpha \beta} p_{\beta} E_{3 \mu \alpha} \\
& \left.\left.-\frac{1}{6}\left(p^{2}+k^{2}\right) E_{3} E_{1} \cdot E_{2}+\text { perms }\right\}\right] .
\end{aligned}
$$

In the limit where the gravitons are nearly on shell, this result coincides with the amplitude $\mathcal{A}$ determined in our scalar theory provided we identify $-6 i M_{\mathrm{P}}^{2}$ with $i N m^{2} /\left(8 \pi^{2} \epsilon\right)$. Hence, we conclude

\footnotetext{
${ }^{5} \mathrm{We}$ would like to warn the attentive reader that the threegraviton vertex as given in Feynman's lectures on gravitation [11] is incomplete. In the literature there are more succinct expressions for the vertex, but they refer to the interactions of another field, namely the tensor density $\mathfrak{g}^{\mu \nu} \equiv \sqrt{-g} g^{\mu \nu}=\eta^{\mu \nu}+\mathfrak{h}^{\mu \nu}$. See for example $[10,12]$.
} 


$$
M_{\mathrm{P}}^{2}=-\frac{N}{48 \pi^{2}} \frac{m^{2}}{\epsilon},
$$

with $\epsilon<0$. Although the $1 / \epsilon$ originates here from a loop diagram in the three-graviton vertex calculation, the resulting relation to $M_{\mathrm{P}}$ agrees with the scattering calculation of Ref. [1], i.e., Eq. (2.13).

We now consider the momentum-independent contributions to the amplitude $\mathcal{A}$, which we denote $\mathcal{A}^{0}$. The contact terms shown in Fig. 3(a) give the contribution

$$
\begin{aligned}
\mathcal{A}_{\text {tree }}^{0}= & -i V_{0}\left[-E_{1} E_{2} E_{3}+2\left(E_{1} E_{2} \cdot E_{3}+\text { perms }\right)\right. \\
& \left.-8 E_{1}^{\mu \nu} E_{2 \nu}{ }^{\alpha} E_{3 \alpha \mu}\right] .
\end{aligned}
$$

The central loop in Fig. 3(b) gives no contribution; this is clear since a momentum-independent part would have to remain in the limit $p^{\mu}=0$, and we have already established that this contribution to $\mathcal{A}$ exclusively involves terms proportional to $p^{\alpha} p^{\beta}$ or $p^{2} \eta^{\alpha \beta}$. On the other hand, we may evaluate the result coming from the diagram of Fig. 2. We find

$$
\begin{aligned}
\mathcal{A}_{\text {loop }}^{0}= & -\frac{1}{2} \frac{i N}{16 \pi^{2}} \frac{m^{4}}{\epsilon}\left[-E_{1} E_{2} E_{3}+2\left(E_{1} E_{2} \cdot E_{3}+\text { perms }\right)\right. \\
& \left.-8 E_{1}^{\mu \nu} E_{2 \nu}{ }^{\alpha} E_{3 \alpha \mu}\right] .
\end{aligned}
$$

Cancellation is assured if

$$
V_{0}=-\frac{N}{2} \frac{m^{4}}{16 \pi^{2} \epsilon},
$$

which is satisfied for the same value of $V_{0}$ that gave us a massless graviton pole. Aside from higher-derivative corrections, our results for the three-graviton coupling are consistent with the expectations of general relativity.

This conclusion is gratifying since the action of our theory is generally covariant and the choice of the background for the clock-and-ruler fields is a convenient gauge choice, analogous to static gauge in string theory. We therefore expect that the form of the three-point coupling should be in accord with general relativity, aside from higher-derivative corrections. Among the sign corrections discussed in Sec. II, we note that the corrected sign in the expression for $V_{0}$ relative to the result in Ref. [1] not only assures the masslessness of the graviton, but here also provides for the nontrivial cancellation between the contact terms in Eq. (4.26), and the one-loop terms in Eq. (4.27).

Finally, we comment on higher-derivative corrections. The diagram in Fig. 2 also contributes to terms in the threegraviton vertex that involve four graviton momenta. Since this part of the central loop is logarithmically divergent, the result is proportional to $N / \epsilon$, which is suppressed by a factor of $m^{2}$ relative to the result of Eq. (4.8). For large $N / \epsilon$, $m$ can be substantially smaller than $M_{\mathrm{P}}$, but still sufficient to render these effects harmless at the distance scales where gravity has been probed [13]. For the sake of argument, if $\epsilon \sim 10^{-11}$, consistent with the phenomenological bound $\epsilon<4 \times 10^{-11}$ from Ref. [14], and $N=100$, one finds $m \approx 7 \times 10^{-6} M_{\mathrm{P}} \approx 10^{13} \mathrm{GeV}$. However, it should be stressed that the suppression of these higher-derivative interactions by $m^{2}$ rather than $M_{\mathrm{P}}^{2}$ is peculiar to dimensional regularization, where the regulator is dimensionless; this result does not generalize to other regulators, for example, Pauli-Villars fields or a Schwinger time / heat kernel UV regulator. We therefore do not consider the suppression of higher-derivative interactions by powers of the scalar mass to be an intrinsic feature of these models.

\section{UNIVERSALITY OF THE GRAVITON COUPLING}

In this section, we consider a theory with two distinct sets of noninteracting scalar fields, $\phi_{1}^{a}$ with $a=1 \ldots N_{1}$, and $\phi_{2}^{b}$ with $b=1 \ldots N_{2}$, distinguished only by their masses $m_{1}$ and $m_{2}$. We wish to show that extending the scalar theory in this way preserves a massless spin-2 graviton state, and that the graviton couples universally to both types of scalars.

The action of the theory, prior to imposing the constraint of vanishing total energy-momentum tensor, is

$$
\begin{aligned}
S= & \int d^{D} x \sqrt{|g|}\left[\frac{1}{2} g^{\mu \nu}\left(\sum_{a=1}^{N_{1}} \partial_{\mu} \phi_{1}^{a} \partial_{\nu} \phi_{1}^{a}+\sum_{a=1}^{N_{2}} \partial_{\mu} \phi_{2}^{a} \partial_{\nu} \phi_{2}^{a}\right)\right. \\
& \left.-\sum_{I, J=0}^{D-1} \partial_{\mu} X^{I} \partial_{\nu} X^{J} \eta_{I J}-V\left(\phi_{1}, \phi_{2}\right)\right] .
\end{aligned}
$$

This extension of the original theory is obtained via the replacements

$\sum_{a=1}^{N} \partial_{\mu} \phi^{a} \partial_{\nu} \phi^{a} \rightarrow \sum_{a=1}^{N_{1}} \partial_{\mu} \phi_{1}^{a} \partial_{\nu} \phi_{1}^{a}+\sum_{a=1}^{N_{2}} \partial_{\mu} \phi_{2}^{a} \partial_{\nu} \phi_{2}^{a}$,

and

$$
V(\phi) \rightarrow V\left(\phi_{1}, \phi_{2}\right) \equiv V_{0}+\Delta V\left(\phi_{1}, \phi_{2}\right),
$$

where

$$
\Delta V\left(\phi_{1}, \phi_{2}\right)=\frac{m_{1}^{2}}{2} \sum_{a=1}^{N_{1}} \phi_{1}^{a} \phi_{1}^{a}+\frac{m_{2}^{2}}{2} \sum_{a=1}^{N_{2}} \phi_{2}^{a} \phi_{2}^{a} .
$$

It follows that

$$
\mathcal{T}^{\mu \nu}=\mathcal{T}_{1}^{\mu \nu}+\mathcal{T}_{2}^{\mu \nu}
$$

The interaction vertex that is relevant to the two-into-two scattering calculation can be inferred from the results of Sec. III 


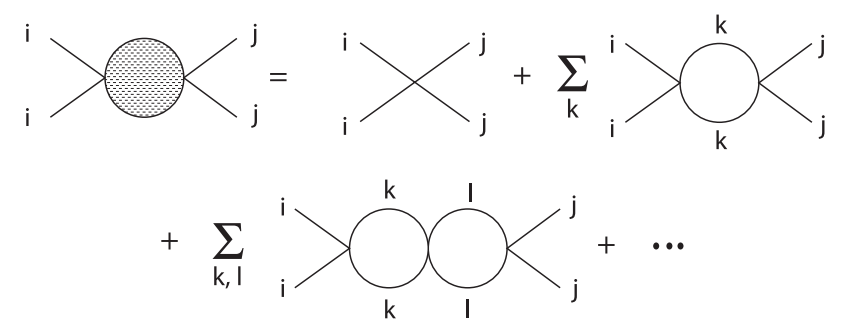

(a)

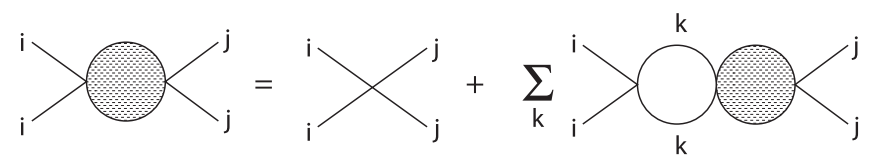

(b)

FIG. 5. Two-into-two scattering diagrams. The labels take the values 1 or 2 , indicating the set of scalars to which a given line is associated; external lines are chosen such that only s-channel diagrams are relevant. Label (b) provides a recursive representation of label (a).

$$
\mathcal{L}_{\text {int }}=-\frac{1}{4 V_{0}} P^{\alpha \beta \mid \mu \nu}\left(\mathcal{T}_{1}+\mathcal{T}_{2}\right)_{\mu \nu}\left(\mathcal{T}_{1}+\mathcal{T}_{2}\right)_{\alpha \beta}
$$

Generalizing our earlier notation, we define the functions

$$
E_{i}^{\mu \nu}(p, k) \equiv-\left(p^{\mu} k^{\nu}+p^{\nu} k^{\mu}\right)+\eta^{\mu \nu}\left(p \cdot k+m_{i}^{2}\right),
$$

for $i=1,2$, corresponding to the Feynman rule for a $\mathcal{T}_{i}$ factor, assuming inwardly or outwardly directed momenta $p$ and $k$. The scattering amplitude shown in Fig. 5(a) has the form

$$
\begin{aligned}
& i \mathcal{M}\left(p_{1}, i ; p_{2}, i \rightarrow p_{3}, j ; p_{4}, j\right) \\
& \quad=E_{i}^{\mu \nu}\left(p_{1}, p_{2}\right)\left[i A_{i j}(q)_{\mu \nu \mid \rho \sigma}\right] E_{j}^{\rho \sigma}\left(p_{3}, p_{4}\right)
\end{aligned}
$$

with $q=p_{1}+p_{2}=p_{3}+p_{4}$. This is equivalent to the recursive representation shown in Fig. 5(b), which leads to

$$
A_{i j}^{\mu \nu \mid \rho \sigma}=A_{0 i j}^{\mu \nu \mid \rho \sigma}+\sum_{k}\left(K_{i k}\right)^{\mu \nu}{ }_{\alpha \beta} A_{k j}^{\alpha \beta \mid \rho \sigma},
$$

where $A_{0}$ corresponds to the tree-level amplitude and we have stripped off the external line factors. It follows from the form of the interaction in Eq. (5.6), however, that the amplitudes $A_{i, j}^{\mu \nu \mid \rho \sigma}$ are independent of $i$ and $j$. Dropping these labels, we may write Eq. (5.9) as

$$
A^{\mu \nu \mid \rho \sigma}=A_{0}^{\mu \nu \mid \rho \sigma}+K_{\alpha \beta}^{\mu \nu} A^{\alpha \beta \mid \rho \sigma},
$$

where

$$
K_{\alpha \beta}^{\mu \nu}=K_{\alpha \beta}^{\mu \nu}\left(N_{1}, m_{1}\right)+K_{\alpha \beta}^{\mu \nu}\left(N_{2}, m_{2}\right),
$$

with the kernel $K^{\mu \nu}{ }_{\alpha \beta}(N, m)$ identical to that of a theory with a single set of $N$ scalar fields with masses $m$ [1],

$$
\begin{aligned}
K_{\alpha \beta}^{\mu \nu}= & -\frac{N(D / 2-1)}{4 V_{0}} \frac{\Gamma(-D / 2)}{(4 \pi)^{D / 2}}\left(m^{2}\right)^{D / 2}\left[1-\frac{D}{12} \frac{q^{2}}{m^{2}}\right] \\
& \times\left(\delta_{\alpha}^{\nu} \delta_{\beta}^{\mu}+\delta_{\beta}^{\nu} \delta_{\alpha}^{\mu}\right)+\mathcal{O}\left(q^{4}\right)
\end{aligned}
$$

where we have included the sign correction noted in Sec. II. The condition that the amplitude in Eq. (5.10) includes a massless pole generalizes to

$$
V_{0}=-\frac{(D / 2-1)}{2} \frac{\Gamma(-D / 2)}{(4 \pi)^{D / 2}} \sum_{i} N_{i}\left(m_{i}^{2}\right)^{D / 2} .
$$

With this tuning of $V_{0}$, one finds

$$
\begin{aligned}
A^{\mu \nu \mid \rho \sigma}= & -\frac{3}{D V_{0}}\left(\frac{\sum_{i} N_{i}\left(m_{i}^{2}\right)^{D / 2}}{\sum_{i} N_{i}\left(m_{i}^{2}\right)^{D / 2-1}}\right) \\
& \times\left[\left(\frac{D}{2}-1\right)\left(\eta^{\nu \rho} \eta^{\mu \sigma}+\eta^{\nu \sigma} \eta^{\mu \rho}\right)-\eta^{\mu \nu} \eta^{\rho \sigma}\right] \frac{1}{q^{2}}+\cdots
\end{aligned}
$$

from which one infers that the Planck mass is given by

$$
M_{\mathrm{P}}=\left[\frac{\Gamma(1-D / 2)}{6(4 \pi)^{D / 2}} \sum_{i} N_{i}\left(m_{i}^{2}\right)^{D / 2-1}\right]^{\frac{1}{D-2}} .
$$

This reduces to the result in the theory with a single set of $N$ scalars in the limit that one or the other of the terms in the sum dominates (or to a theory of $2 N$ scalars of mass $m$, when $m_{1}=m_{2}=m$ ). Note that for comparable $N_{1}$ and $N_{2}$, the Planck scale is set by the mass of heavier of the scalars, while the other can be much lighter.

In the two-scalar theory, the metric fluctuation that follows from the constraint of vanishing energy-momentum tensor can be written

$$
\begin{aligned}
h_{\mu \nu}= & \frac{D / 2-1}{V_{0}}\left[\sum_{a=1}^{N_{1}} \partial_{\mu} \phi_{1}^{a} \partial_{\nu} \phi_{1}^{a}+\sum_{a=1}^{N_{2}} \partial_{\mu} \phi_{2}^{a} \partial_{\nu} \phi_{2}^{a}\right] \\
& -\eta_{\mu \nu} \frac{\Delta V}{V_{0}}+\mathcal{O}\left(1 / V_{0}^{2}\right),
\end{aligned}
$$

which is algebraically equivalent to

$$
h_{\mu \nu}=\frac{1}{V_{0}} P_{\mu \nu}^{\alpha \beta}\left(\mathcal{T}_{1}+\mathcal{T}_{2}\right)_{\alpha \beta}+\mathcal{O}\left(1 / V_{0}^{2}\right) .
$$

Following the same approach we used previously to write down the lowest-order effective interaction for a fundamental graviton field, we find from Eq. (5.6),

$$
\mathcal{L}_{\text {eff }}=-\frac{1}{2} h_{\mu \nu}\left(\mathcal{T}_{1}+\mathcal{T}_{2}\right)^{\mu \nu}
$$

where we have taken into account the two ways in which we may identify the fundamental graviton field. This is of 
the expected form, consistent with a universal coupling of the graviton to the two types of scalar fields in the theory. The strength of the gravitational interaction is set by $M_{\mathrm{P}}$ in Eq. (5.15). The results of this section extend trivially to more than two sets of distinct scalar fields.

\section{THE EXPECTATION VALUE OF THE EMERGENT METRIC}

In the current setup, where the energy-momentum tensor has a vanishing vacuum expectation value (VEV), the expectation value of the metric fluctuation

$$
h_{\mu \nu}^{(1)}=\frac{1}{V_{0}} P_{\mu \nu \mid \alpha \beta} \mathcal{T}^{\alpha \beta}
$$

is zero, and the metric is flat.

We would like to consider a scenario where a conserved source term is added to the action,

$$
\delta \mathcal{S}=-\frac{1}{2} \int d^{D} x J^{\alpha \beta}(x) P_{\alpha \beta \mid \mu \nu} \mathcal{T}^{\mu \nu} .
$$

In the presence of the external source, $J_{\alpha \beta}$, the metric fluctuation can acquire a non-zero VEV

$$
\left\langle h_{\mu \nu}^{(1)}\right\rangle_{J}=\frac{1}{Z_{J}} \int \mathcal{D} \phi h_{\mu \nu}^{(1)} e^{i(\mathcal{S}[\phi]+\delta \mathcal{S})},
$$

where the normalization factor $Z_{J}$ is the partition function in the presence of the source. Diagrammatically, the VEV is given by an infinite sum of which the lowest order terms are depicted in Fig. 6.

This procedure mirrors at each step the general relativity calculation performed by Duff [10] who showed that by summing such quantum tree diagrams, where the wavy lines in Fig. 6 denote the flat space graviton propagator, one can recover, for example, an order-by-order (in terms of mass) expansion of the Schwarzschild metric, if the source is taken to be spherically symmetric. By analogy, we expect

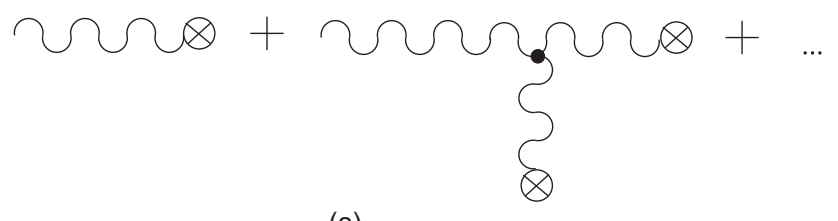

(a)

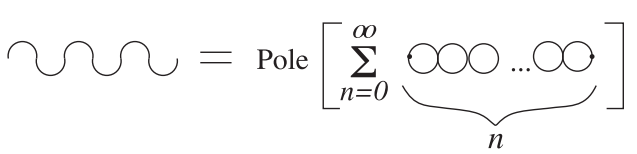

(b)

FIG. 6. (a) The Feynman diagrams contributing to the VEV of the metric fluctuation in the presence of an external source. The source is depicted by the crossed circle. The second diagram includes the three-graviton vertex. (b) Scalar loops that lead to the emergent graviton pole. the same result to hold in our theory, so that we obtain an emergent curved metric. ${ }^{6}$

\section{CONCLUSIONS}

In this paper, we have considered graviton interactions in a theory of emergent gravity proposed by Carone, Erlich and Vaman [1]. The original proposal was a theory of $D+N$ scalar fields and was defined via a functional integral, subject to the constraint of vanishing total energy-momentum tensor. The set of $D$ fields, called clock-and-ruler fields, had profiles that determined the background metric, but otherwise could be gauged away. The composite graviton state was found to couple to the nonvanishing energy-momentum tensor of the remaining $N$ scalar fields. While the original work in Ref. [1] demonstrated the existence of a massless, spin-2 pole in scalar two-into-two scattering amplitudes in the large $N$ limit, the coupling of the graviton to itself, the universality of the coupling when additional scalars of differing mass are present, and an approach to incorporating curved backgrounds were not discussed. Those issue were clarified in the present work.

In particular, we showed here that the effective threegraviton coupling inferred from scattering amplitudes involving six external scalar lines is consistent with the expectations of the weak-field limit of general relativity. The internal graviton lines in the theory with a fundamental graviton field are contained within sums over loop subdiagrams that each generate an emergent graviton pole. The scattering amplitude arises by connecting these loop chains using the available four- and six-scalar vertices in the theory. This is similar to the way that non-Abelian gauge boson vertices were generated in the composite models of Ref. [9]. We showed by direct calculation that the Planck scale inferred from the induced three-graviton vertex, a function of the generally covariant physical regulator of the theory, agrees with the result found in the scattering calculation of Ref. [1]. Moreover, we explained in the present work why the perturbative expansion about a fixed background does not lead to an explicit breaking of general covariance, once loop corrections are taken into account.

The success of our emergent gravity model in reproducing the Einstein-Hilbert three-graviton coupling is predicated on starting with the diffeomorphism-invariant scalar action given in Eq. (2.6). At the same time, for those readers familiar with Sakharov's model for induced gravity, it might be worth pointing out that our Fig. 2 Feynman diagram bears a resemblance with the matter one-loop Feynman diagram which induces the cubic graviton two-derivativecoupling interaction terms in Sahkarov's model. Therefore it

\footnotetext{
${ }^{6}$ For a different approach to incorporating curved backgrounds in the present framework, and which involves a modification of the starting point in Ref. [1], see Ref. [15].
} 
might seem that we are guaranteed in recovering the EinsteinHilbert cubic graviton interaction. However, in Sakharov's model, the matter loops also induce nonvanishing cubic graviton interaction with no derivatives, and which can be interpreted as arising from a cubic order expansion of a cosmological constant term. Such terms are absent in our approach since we have canceled the cosmological constant by construction. Specifically, the fine tuning given in Eq. (2.10) which ensured the existence of the massless composite graviton also led to the vanishing of the nonderivative cubic graviton interaction terms as detailed in Eqs. (4.26) and (4.27).

We also considered extending the theory to the case where we begin with two sets of noninteracting scalar fields of differing masses. This is a natural consistency check on the theory, since it would be unacceptable if, for example, there were a different effective Planck mass for each set of scalars. We verified that the composite graviton couples to the energy-momentum tensor of each set of scalars with a strength set by a single Planck mass, which is a function of the number and mass of each type of scalar field. We noted that in the case where there is a hierarchy in the scalar masses (assuming the numbers of each type are comparable), the Planck scale is determined by the heavier scalar, while the other can be arbitrarily lighter. Finally, we briefly noted how to incorporate the physics of a curved background while still expanding about a flat one, by coupling the composite scalar operator that is identified with the metric to a classical source, implementing an approach known in the context of the weak-field limit of general relativity.

A number of issues are appropriate for future work. In the type of scalar theories considered here, it is worthwhile considering how the diagrammatic analysis generalizes in the case where scalar interactions are present in the theory before the constraint of vanishing energy-momentum tensor is applied. More precisely, how would the analysis be modified if the scalar potential in Eq. (2.9) were to include, for example, a quartic term? While the diagrams included in the scattering calculation of Ref. [1] are still present, additional diagrams that contribute to the amplitude are expected; it needs to be shown that the graviton pole persists in this case. Another interesting issue is the generalization of the diagrammatic approach of Ref. [1] to theories involving fields of spin- $1 / 2$ and 1 , required for applications to phenomenologically relevant quantum field theories. Work in that direction is underway [16], and will be presented elsewhere.

\section{ACKNOWLEDGMENTS}

The authors are grateful for many fruitful discussions with Joshua Erlich. The work of C. D. C. and T. V. B. C. was supported by the NSF under Grant No. PHY-1519644. The work of D. V. was supported in part by DOE Grant No. DE-SC0007894. D. V. would also like to acknowledge the hospitality of the College of William and Mary Physics Department for the duration of this work.

\section{APPENDIX: THREE-GRAVITON VERTEX DECOMPOSITION}

The Feynman rule for the three-graviton vertex in the weak-field expansion of general relativity was used in Sec. IV. One can understand the Feynman rule from the following considerations: The three-graviton part of $\sqrt{g} R$ can be decomposed in terms of an operator basis, where each operator involves two derivatives and three graviton fields. The following 14 operators form a suitable basis:

$$
\begin{aligned}
O_{1} & =h^{2} \square h \\
O_{2} & =h \square h^{\alpha}{ }_{\beta} h^{\beta}{ }_{\alpha} \\
O_{3} & =h \partial_{\mu} h^{\alpha}{ }_{\beta} \partial^{\mu} h^{\beta}{ }_{\alpha} \\
O_{4} & =h^{\alpha}{ }_{\beta} \square h^{\beta}{ }_{\mu} h^{\mu}{ }_{\alpha} \\
O_{5} & =h_{\mu \nu} \partial^{\mu} h \partial^{\nu} h \\
O_{6} & =h_{\mu \nu} h \partial^{\mu} \partial^{\nu} h \\
O_{7} & =h_{\mu \nu} \partial^{\mu} h^{\alpha}{ }_{\beta} \partial^{\nu} h^{\beta}{ }_{\alpha} \\
O_{8} & =h_{\mu \nu} h^{\alpha}{ }_{\beta} \partial^{\mu} \partial^{\nu} h^{\beta}{ }_{\alpha} \\
O_{9} & =h \partial^{\mu} h_{\mu \alpha} \partial^{\nu} h_{\nu}{ }^{\alpha} \\
O_{10} & =h \partial^{\mu} \partial^{\nu} h_{\mu \alpha} h_{\nu}{ }^{\alpha} \\
O_{11} & =h \partial^{\nu} h_{\mu \alpha} \partial^{\mu} h_{\nu}{ }^{\alpha} \\
O_{12} & =h^{\alpha \beta} \partial^{\mu} h_{\mu \alpha} \partial^{\nu} h_{\nu \beta} \\
O_{13} & =h^{\alpha \beta} \partial^{\mu} \partial^{\nu} h_{\mu \alpha} h_{\nu \beta} \\
O_{14} & =h^{\alpha \beta} \partial^{\nu} h_{\mu \alpha} \partial^{\mu} h_{\nu \beta} .
\end{aligned}
$$

The cubic terms of $\sqrt{g} R$ can then be written

$$
\begin{aligned}
{[\sqrt{g} R]^{(3)}=} & {\left[\frac{1}{16} O_{1}-\frac{1}{2} O_{2}-\frac{3}{8} O_{3}+\frac{1}{4} O_{4}\right] } \\
& +\left[-\frac{1}{4} O_{6}+\frac{1}{4} O_{7}+\frac{1}{2} O_{8}\right] \\
& +\left[\frac{1}{2} O_{9}+O_{10}+\frac{1}{4} O_{11}-O_{13}-\frac{1}{2} O_{14}\right]
\end{aligned}
$$

The momentum-space Feynman rule that follows from Eq. (A15) leads to the quantity in parentheses in Eq. (4.22). The prefactor of $4 / M_{\mathrm{P}}$ arises from the following considerations: we start with the Lagrangian $\mathcal{L}=$ $-M_{\mathrm{P}}^{2}[\sqrt{g} R] / 2+h_{\mu \nu} \mathcal{T}^{\mu \nu} / 2$, and then rescale $h_{\mu \nu} \rightarrow 2 h_{\mu \nu} /$ $M_{\mathrm{P}}$ to place the graviton kinetic terms in canonical form. This leads to the graviton-matter coupling $h_{\mu \nu} \mathcal{T}^{\mu \nu} / M_{\mathrm{P}}$, mentioned in Sec. IV, as well as an overall factor of $-4 / M_{\mathrm{P}}$ multiplying the Feynman rule that follows from Eq. (A15). 
[1] C. D. Carone, J. Erlich, and D. Vaman, Emergent gravity from vanishing energy-momentum tensor, J. High Energy Phys. 03 (2017) 134.

[2] S. Weinberg and E. Witten, Limits on massless particles, Phys. Lett. B 96, 59 (1980).

[3] A. D. Sakharov, Vacuum quantum fluctuations in curved space and the theory of gravitation, Sov. Phys. Dokl. 12, 1040 (1968); Dokl. Akad. Nauk Ser. Fiz. 177, 70 (1967); Sov. Phys. Usp. 34, 394 (1991); Gen. Relativ. Gravit. 32, 365 (2000).

[4] M. Visser, Sakharov's induced gravity: A modern perspective, Mod. Phys. Lett. A 17, 977 (2002).

[5] E. P. Verlinde, Emergent gravity and the dark universe, SciPost Phys. 2, 016 (2017); On the origin of gravity and the laws of Newton, J. High Energy Phys. 04 (2011) 029.

[6] H. C. Ohanian, Gravitons as goldstone bosons, Phys. Rev. 184, 1305 (1969).

[7] L. Sindoni, Emergent models for gravity: An overview of microscopic models, SIGMA 8, 027 (2012).

[8] H. Terazawa, K. Akama, and Y. Chikashige, Unified model of the Nambu-Jona-Lasinio type for all elementary particle forces, Phys. Rev. D 15, 480 (1977); K. Akama, Y. Chikashige, T. Matsuki, and H. Terazawa, Gravity and electromagnetism as collective phenomena: A derivation of Einstein's general relativity, Prog. Theor. Phys. 60, 868 (1978); K. Akama, An attempt at pregeometry: Gravity with composite metric, Prog. Theor. Phys. 60, 1900 (1978); D. Amati and G. Veneziano, Metric from matter, Phys. Lett. 105B, 358 (1981); A unified gauge and gravity theory with only matter fields, Nucl. Phys. B204, 451 (1982); K. Akama and T. Hattori, Dynamical foundations of the brane induced gravity, Classical Quantum Gravity 30, 205002 (2013).

[9] M. Suzuki, Composite gauge-bosons made of fermions, Phys. Rev. D 94, 025010 (2016); Inevitable emergence of composite gauge bosons, Phys. Rev. D 96, 065010 (2017).

[10] M. J. Duff, Quantum tree graphs and the Schwarzschild solution, Phys. Rev. D 7, 2317 (1973).

[11] R. P. Feynman, F. B. Morinigo, and W. G. Wagner, Feynman Lectures on Gravitation (Addison-Wesley, Reading, MA, 1995).

[12] J. N. Goldberg, Conservation laws in general relativity, Phys. Rev. 111, 315 (1958).

[13] See Experimental tests of gravitational theory, in C. Patrignani et al. (Particle Data Group), Review of particle physics, Chin. Phys. C 40, 100001 (2016).

[14] A. Schafer and B. Muller, Bounds for the fractal dimension of space, J. Phys. A 19, 3891 (1986).

[15] S. Chaurasia, J. Erlich, and Y. Zhou, Curved backgrounds in emergent gravity, Classical Quantum Gravity 35, 115008 (2018)..

[16] C. D. Carone, J. Erlich, and D. Vaman (in preparation). 